\title{
Spinon-orbiton repulsion and attraction mediated by Hund's rule
}

\author{
Jonas Heverhagen ${ }^{1,2}$ and Maria Daghofer ${ }^{1,2}$ \\ ${ }^{1}$ Institute for Functional Matter and Quantum Technologies, \\ University of Stuttgart, Pfaffenwaldring 57 D-70550 Stuttgart, Germany \\ ${ }^{2}$ Center for Integrated Quantum Science and Technology, \\ University of Stuttgart, Pfaffenwaldring 57 D-70550 Stuttgart, Germany
}

(Dated: April 13, 2018)

\begin{abstract}
We study the impact of Hund's-rule coupling on orbital excitations, as e.g. measured in inelastic resonant $\mathrm{x}$-ray scattering. We find that the interpretation in terms of spin-orbit separation, which has been derived for one-dimensional systems without Hund's rule, remains robust in its presence. Depending on whether or not the orbital excitation includes a spin excitation, Hund's rule leads to an attractive or repulsive interaction between spinon and orbiton. Attraction and repulsion leave clear signatures through a transfer of spectral weight to the lower resp. upper edge of the spectrum.
\end{abstract}

PACS numbers: 75.25.Dk,75.30.Ds,75.10.Jm,75.10.Pq

Introduction. Low-dimensional quantum systems have long been of special interest due to the intriguing and often counter-intuitive properties they can host when quantum fluctuations and interactions come together. Arguably the strangest concept at play is fractionalization, where the electron, an elementary particle, behaves as if it were split into parts with fractional charges (e.g. in two-dimensional fractional quantumHall states) or into a charge separated from its spin [1]. This last concept, spin-charge separation, applies to an electron or hole propagating in a one-dimensional Mott insulator. Spin and charge can then be considered as propagating as 'spinon' and 'holon' with different velocities, as has been studied theoretically [2, 3, and verified experimentally using angle-resolved photo-emission spectroscopy [4, 5].

More recently, spin-charge separation has been complemented by the idea of spin-orbit separation [6]. This also occurs in one-dimensional Mott insulators, but involves an electron being excited into some unoccupied higherenergy orbital instead of being removed from the system. As has been pointed out theoretically, the orbital excitation can then be considered in a manner analogous to a hole and similarly separates into 'spinon' and 'orbiton'. Experimentally, this has been verified using resonant inelastic X-ray scattering (RIXS), which can address orbital excitations, in a cuprate chain compound [7.

The theory behind the analogy of spin-orbit and spincharge separation rests on a mapping [6] of the orbital excitation onto a hole-removal excitation that, strictly speaking, breaks down in the presence of Hund's-rule coupling [8]. While it seems reasonable to assume that small Hund's-rule coupling should not completely invalidate the scenario, the question of its impact remains open. As it must be assumed to be present in any realistic material scenario, we want to assess how far the mapping and the scenario of spin-orbit separation can be trusted.

We show in this letter that an interpretation in terms of spinon and orbiton survives to a very large degree and that the main effect of Hund's-rule coupling is an interaction between spinon and orbiton. The issue of spinonholon interaction in the $t$ - $J$ model has been discussed analytically in the supersymmetric limit 9, where some exact results can be obtained, and turned out to be rather subtle [10, 11. Numerically, spinon-holon attraction has been followed from the $t-J^{z}$ model, where it leads to a bound state, to the isotropic $t-J$ model, where it was concluded to be present but too weak for a bound state [12] The present work indicates that orbital excitations provide an intriguing window into the interactions between fractionalized excitations: they can address the repulsive as well as the attractive regime and for strong Hund'srule coupling, signatures of spinon-orbiton interaction become quite pronounced.

Orbital excitations in antiferromagnets. We consider here two orbitals per site, denoted by 1 for the low-energy and 2 for the high-energy state, and the limit of strong onsite Coulomb repulsion $U$, i.e., we neglect charge fluctuations. Second order perturbation theory with intersite hopping $t$ as a small parameter $t / U$ then gives a KugelKhomskii-type model [13] with the general form

$$
H=2 \sum_{\langle i, j\rangle}\left(\vec{S}_{i} \cdot \vec{S}_{j}+\frac{1}{4}\right) A_{i j}+\sum_{\langle i, j\rangle} K_{i j}+\Delta \sum_{i} T_{i}^{z},
$$

where $\vec{S}_{i}$ describes a spin $S=\frac{1}{2}$ at site $i$ and $T_{i}^{z}=$ $\frac{1}{2}\left(n_{2}-n_{1}\right)$ is the $z$ component of the orbital pseudospin. Operators $A_{i j}$ and $K_{i j}$ depend on the orbital degrees of freedom, see below. Bonds $\langle i, j\rangle$ run over nearest neighbors $(\mathrm{NN})$, but can be chosen to include longer-range interactions. Strong crystal field $\Delta \gg t$ ensures that only lower-energy orbital 1 is occupied in the ground state.

Since states with two electrons on one site enter the perturbation theory as (virtual) intermediate states, $A_{i j}$ and $K_{i j}$ depend on the onsite interactions. It is helpful to first consider any doubly occupied site to have energy $U$, regardless of its spin and orbital occupation. This 
neglects processes like Hund's-rule coupling but brings out the dominant terms

$$
A_{i j}^{(0)}=\frac{4 t^{2}}{U}\left[\left(T_{i}^{z} T_{j}^{z}+\frac{1}{4}\right)+\frac{1}{2}\left(T_{i}^{+} T_{j}^{-}+\text {H.c. }\right)\right], K_{i j}^{(0)}=0 .
$$

(We assume identical hopping $t$ in both orbitals.) When strong crystal field splitting $\Delta$ enforces orbital polarization $T_{i}^{z} \equiv-\frac{1}{2}$, the second term in $A_{i j}^{(0)}$ is inactive in the ground state, and the first term leads to antiferromagnetic (AFM) Heisenberg coupling of the spins in (1).

An orbital excitation is then induced into the AFM state, e.g. by RIXS, which allows for this excitation to come with or without a spin flip. The two excitations can be distinguished in experiment [14, 15] and are described by operators

$$
\begin{aligned}
T^{+}(k) & =\frac{1}{\sqrt{L}} \sum_{j, \sigma} \mathrm{e}^{i k j} c_{j, 2, \sigma}^{\dagger} c_{j, 1, \sigma} \quad \text { and } \\
\left(S^{x} T^{+}\right)(k) & =\frac{1}{\sqrt{L}} \sum_{j, \sigma} \mathrm{e}^{i k j} c_{j, 2,-\sigma}^{\dagger} c_{j, 1, \sigma}
\end{aligned}
$$

where $c_{j, 2, \sigma}^{\dagger}\left(c_{j, 1, \sigma}\right)$ creates (annihilates) an electron with spin $\sigma= \pm 1=\uparrow, \downarrow$ in the empty orbital 2 (occupied orbital 1 ) on site $j . k$ denotes crystal momentum and runs over the first Brillouin zone of the $L$-site chain.

The excitations move via the second term in $A_{i j}^{(0)}$, see Eq. (2), and it turns out that the spin in the upper orbital 2 is conserved and has no impact on either kinetic or potential energy [6, 16. Excitations with and without spin flip are thus equivalent and can be mapped onto a spinless hole moving in an AFM background. This mapping between orbital excitations and hole dynamics has been used extensively to analyze RIXS in onedimensional cuprates [7, 17, 18] and two-dimensional iridates [16, 19, 20].

Richer structure of the doubly occupied site. While the dominant terms discussed above are helpful and illustrative, a realistic description of two electrons on one site has to take into account processes beyond pure charge interactions. Let $U$ denote Coulomb repulsion felt by two electrons in the same orbital on the same site $i$. Due to reduced overlap of the wave functions, their interaction $U^{\prime}=U-\Delta U<U$ is weaker if they occupy different orbitals. In that case, Hund's-rule coupling $-2 J_{H} \vec{S}_{i, 1} \vec{S}_{i, 2}$ moreover favors their ferromagnetic (FM) alignment. Finally, a 'pair hopping' $J_{P}$ involves a doubly occupied high-energy orbital 2 and is suppressed here by the large crystal field. The full Kugel-Khomskii Hamiltonian is given by

$$
\begin{aligned}
& A_{i j}=\frac{4 U t^{2}}{U^{2}-J_{P}^{2}}\left(T_{i}^{z} T_{j}^{z}+\frac{1}{4}\right)+\frac{4 J_{H} t^{2}}{U^{\prime 2}-J_{H}^{2}}\left(T_{i}^{z} T_{j}^{z}-\frac{1}{4}\right)+ \\
& +\frac{2 U^{\prime} t^{2}}{U^{\prime 2}-J_{H}^{2}}\left(T_{i}^{+} T_{j}^{-}+\text {H.c. }\right)-\frac{2 J_{P} t^{2}}{U^{2}-J_{P}^{2}}\left(T_{i}^{-} T_{j}^{-}+\text {H.c. }\right)
\end{aligned}
$$

and

$$
\begin{aligned}
& K_{i j}=-\frac{4 U t^{2}}{U^{2}-J_{P}^{2}}\left(T_{i}^{z} T_{j}^{z}+\frac{1}{4}\right)+\frac{4 U^{\prime} t^{2}}{U^{\prime 2}-J_{H}^{2}}\left(T_{i}^{z} T_{j}^{z}-\frac{1}{4}\right)+ \\
& +\frac{2 J_{H} t^{2}}{U^{\prime 2}-J_{H}^{2}}\left(T_{i}^{+} T_{j}^{-}+\text {H.c. }\right)+\frac{2 J_{P} t^{2}}{U^{2}-J_{P}^{2}}\left(T_{i}^{-} T_{j}^{-}+\text {H.c. }\right) .
\end{aligned}
$$

We use here relations $J_{H}=J_{P}$ and $\Delta U=2 J_{H}$, which arise naturally for symmetry-related orbitals [24, 25, but have checked that deviations do not significantly alter our results.

We apply (Lanczos) exact diagonalization to Hamiltonian (1) with orbital operators (5)-(6). To reach longer chains, only states with at most one electron in the higher-energy orbital 2 are kept, which does not affect results in our limit of large crystal-field splitting. Exact diagonalization is complemented with spin-clusterperturbation theory 26, which gives limited access to momentum points not directly available on the directly solved cluster and which has been previously applied to orbital excitations [8].

Numerical results and spinon-orbiton interaction. Figure 1 shows spectra for excitations (3) and (4) without and with a spin flip, for increasing deviation from the high-symmetry case (2). At small $J_{H} / U=1 / 20$, excitations with and without spin flip look nearly identical, see Figs. 1(a) and 1(b) They also strongly resemble the $J_{H}=0$ result [6], which in turn correspond to the one-particle spectral density of the supersymmetric $t-J$ model [27] with $t=J / 2$. The spectrum can then be described in terms of spinon and holon interacting via a phase string [23, where the role of the holon is here taken by the 'orbiton'. The lens-shaped dominant feature can thus be identified with the one-spinon-one-holon (orbiton) part of the spectrum, while the additional small weight at higher energy towards $k=\pi$ comes from states with three spinons [21, 22].

At larger $J_{H} / U=3 / 20$, the lens can still be recognized, albeit with a broadened energy range. However, spectral weight has clearly shifted to its high-energy (low-energy) edge for the pure orbital (combined spinorbital) excitation, see Figs. 1(c) and 1(d) Finally at $J_{H} / U=5 / 20=1 / 4$, energy range has further increased and spectral weight is almost completely located on the upper (lower) side without (with) a spin flip. For the combined spin-orbital excitation, Fig. 1(f) shows features like the 'spinon' and 'holon' branches familiar from the $t$ - $J$ model, however, the 'holon' is broadened.

In order to interpret the features and understand their origin, corrections to 2 in first-order of $\frac{1}{U}$ can be analyzed. The part $K_{i j}$ decoupled from spins no longer 

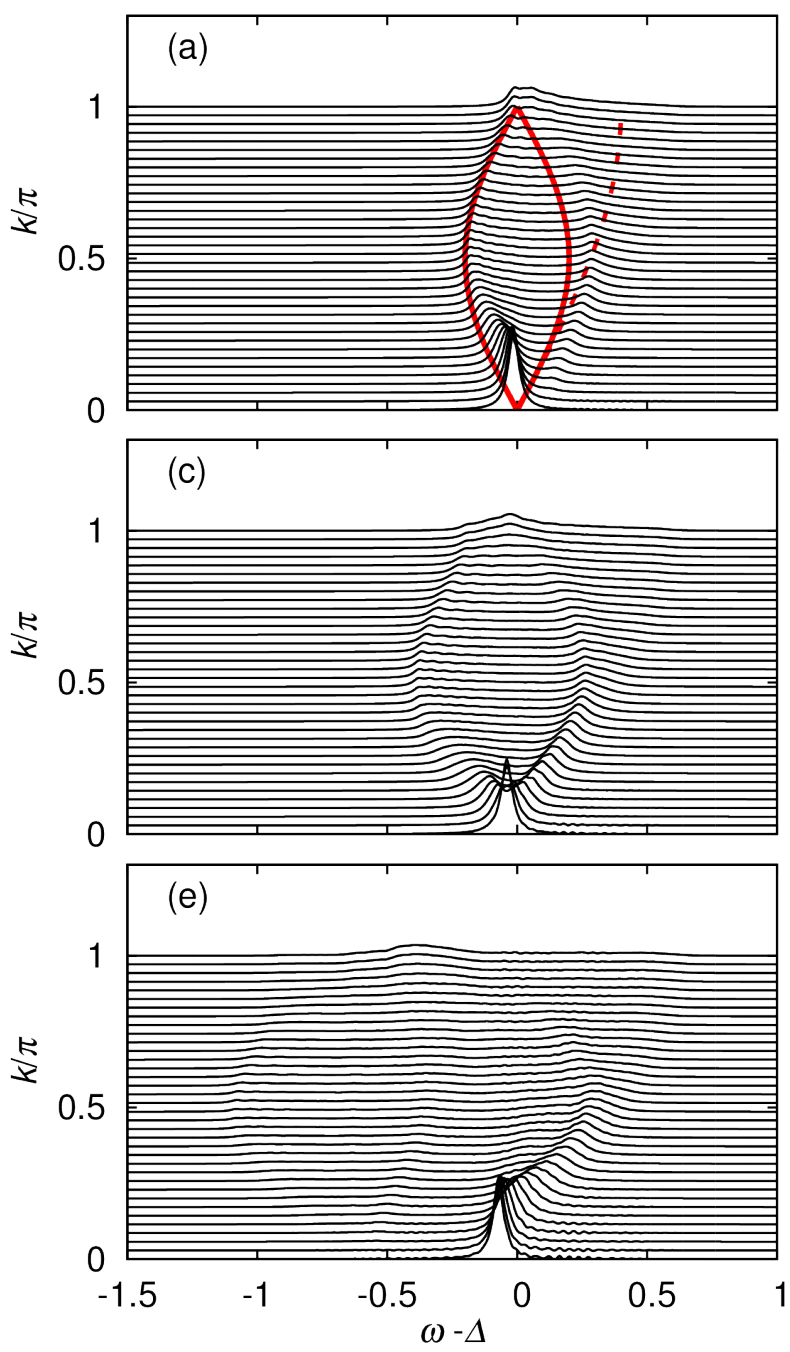
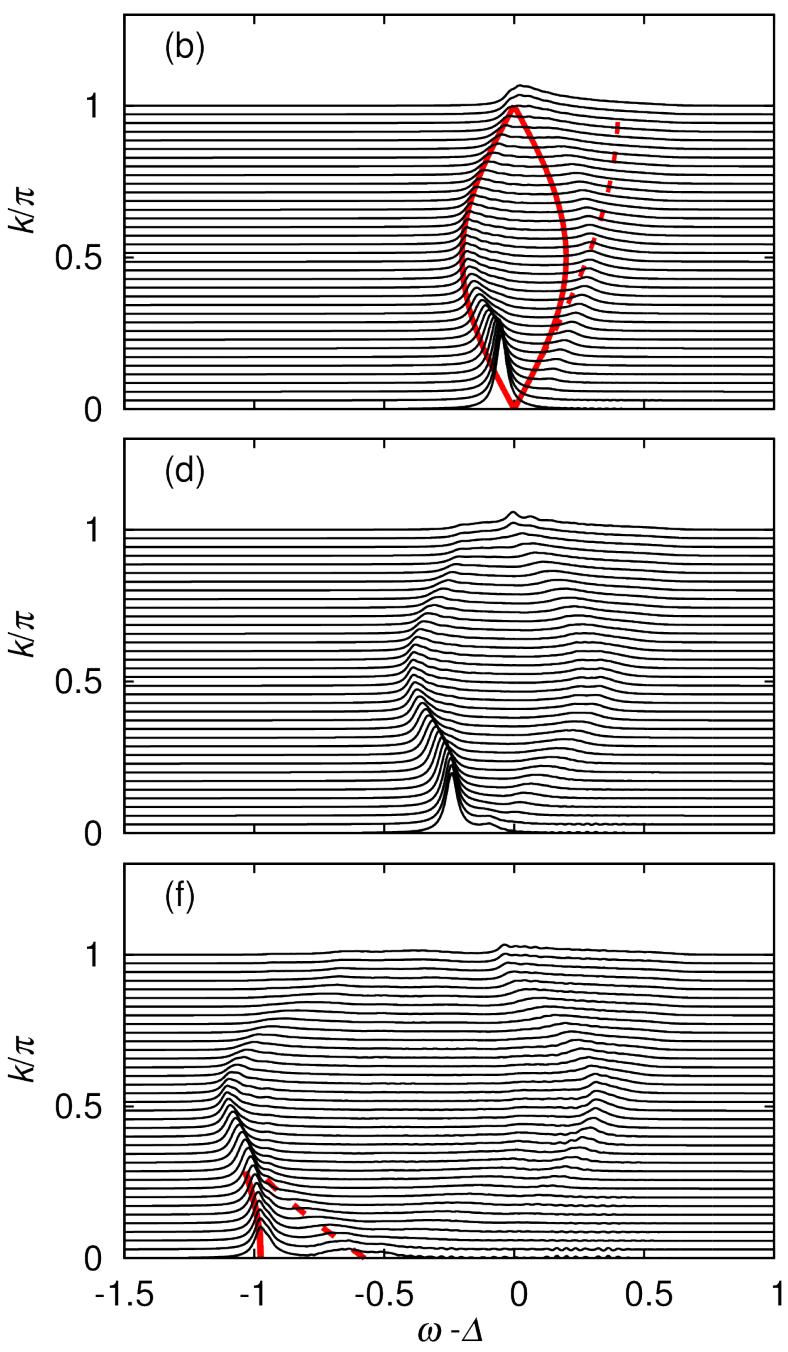

FIG. 1. Orbital excitations with increasing $J_{H}=J_{P}=\Delta U / 2$. Spectra for the pure orbital excitation Eq. (3) without a spin flip are shown in the left column for (a) $J_{H}=t=U / 20,(\mathrm{c}) J_{H}=3 t=3 U / 20$, and (e) $J_{H}=5 t=U / 4$. The right column gives spectra for the spin-orbital excitation Eq. (4), which includes a spin flip, for (b) $\left.J_{H}=t,(\mathrm{~d})\right] J_{H}=3 t$, and (f) $J_{H}=5 t$. The broad solid (dashed) lines in (a) and (b) give the approximate support of the one-spinon-one-holon (three-spinon-one-holon) part of the one-particle spectrum of the $t-J$ model 21, 22. This support is consistent with a phenomenological analysis in terms of spinon and holon interacting via a phase string [23] and corresponds to the orbital excitation spectrum for $J_{H}=0$. In (f)] the broad solid and dashed lines are guides to the eye following the peaks of the branches identified as 'spinon' and 'orbiton' branches. Results obtained with spin CPT based on $L=24$ sites, Coulomb repulsion $U=20 t$, crystal field $\Delta=10 t$.

vanishes,

$$
\begin{aligned}
K_{i j}^{(1)}= & \frac{4 t^{2}}{U}\left[\frac{\Delta U}{U}\left(T_{i}^{z} T_{j}^{z}-\frac{1}{4}\right)+\frac{J_{H}}{2 U}\left(T_{i}^{+} T_{j}^{-}+\text {H.c. }\right)\right. \\
& \left.+\frac{J_{P}}{2 U}\left(T_{i}^{-} T_{j}^{-}+\text {H.c. }\right)\right]
\end{aligned}
$$

where the last term $\propto J_{P}$ is suppressed by crystal-field splitting $\Delta$ and the first term $\propto \Delta U$ gives a small overall energy shift. The second term $\propto J_{H}$ allows the excited orbital to move without an onsite spin flip, so that the spin of the excitation can flip. In the $1 \mathrm{D}$ chain, this can induce additional spinons and is a likely reason for, e.g., the broadening of the 'holon' branch in Fig. 1(f). Despite its rather minor role here, we expect this term to have a more decisive effect in higher dimensions, where it would allow the orbital excitation to travel 'freely' through an AFM ordered state without creating a string potential.

Corrections to $A_{i j}$ are

$$
\begin{aligned}
A_{i j}^{(1)}= & \frac{4 t^{2}}{U}\left[\frac{J_{H}}{U}\left(T_{i}^{z} T_{j}^{z}-\frac{1}{4}\right)+\frac{\Delta U}{2 U}\left(T_{i}^{+} T_{j}^{-}+\text {H.c. }\right)\right. \\
& \left.-\frac{J_{P}}{2 U}\left(T_{i}^{-} T_{j}^{-}+\text {H.c. }\right)\right]
\end{aligned}
$$

where the last term is again suppressed. The second terms here and in Eq. (2) have exactly the same form and same sign, so that the main effect of $U^{\prime}<U$ is to increase orbiton hopping relative to spin superexchange [16. This in turn increases band width and makes the orbiton faster 


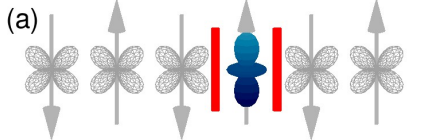

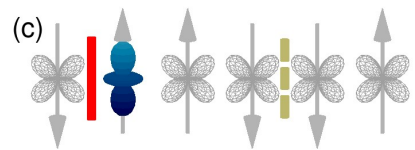
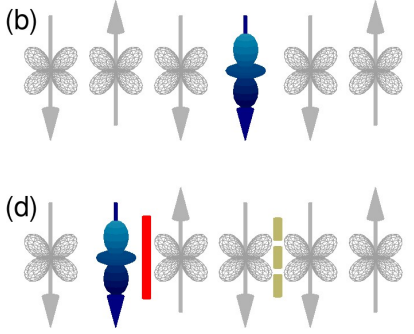

FIG. 2. Cartoon of the effective spinon-orbiton repulsion/attraction. In (a) and (b), orbital excitations without/with spin flip are created, spinon and orbiton are located on the same spot. In (c) and (d) the orbiton has moved two sites, leaving behind the spinon. Broken vertical bars between sites indicate spinons; solid bars violations of GoodenoughKanamori rules on bonds with alternating orbitals.

than the spinon, so that we recover the 'usual' spinonholon scenario in Fig. 1(f),

Finally, the first term of $(8)$ becomes negative between two sites with different orbital occupation because $T_{i}^{z} T_{j}^{z}=-\frac{1}{4}$ in that case, while the term vanishes for identical orbitals and $T_{i}^{z} T_{j}^{z}=+\frac{1}{4}$. Negative $A_{i j}$ implies FM spin-spin coupling in the Kugel-Khomskii Hamiltonian (1). This is opposite to the AFM coupling between identical orbitals that comes from the first term of (2), which in turn vanishes between sites with different orbital occupation. The sign change of the magnetic interaction driven by different orbital occupation, known as Goodenough-Kanamori rules [28, 29, often contributes to complex magnetic orderings in the presence of orbital degrees of freedom. We argue here that this effect also mediates an interaction between the excited orbital and the spinon, see Fig. 2, that plays out differently for the excitations with and without a spin flip.

In (short-range) AFM order, the excited electron after a process Eq. (4) with spin flip has the same spin as its two neighbors in lower-energy orbitals, see Fig. 2(b)] exactly the situation energetically favored by the first term of (8). If the excited orbital (i.e. the orbiton) moves away, leaving behind a domain wall (i.e. the spinon), it is generally found between spins of opposite sign, so that only one bond can profit from FM exchange. The other bond, indicated by a red vertical bar in Fig. 2(d), is AFM and raises the energy for separating spinon and orbiton; orbiton and spinon thus see an attractive potential. In contrast, an orbital excited without a spin flip in process Eq. (3) has spin opposite to its two neighbors, see Fig. 2(a) and both bonds pay energy. Separated from the spinon and sitting between an up and a down spin, it has spin parallel to one of its neighbors, see Fig. 2(d), which reduces energy cost. Accordingly, spinon and orbiton repel each other here.

However, the cartoon Fig. 2 with its perfectly ordered Ising spins overemphasizes spinon-orbiton attrac- tion, because it suggests that the domain wall costs energy $\propto J=\frac{4 t^{2}}{U}$ anywhere except at the site of the orbiton. This interaction, indicated by broken vertical bars in Fig. 2. would be independent of $J_{H}$ and indeed binds spinon and holon together in the $t-J^{z}$ model [30. While the effect has been found too weak to induce a bound state in the spin-isotropic $t-J$ model with its half-filled spinon-sea groundstate, it is sizable on finite chains [12].

Fortunately, Hund's-rule-driven spinon-orbiton interaction can be distinguished from this 'baseline' interaction even on small systems by use of open boundary conditions (OBC). To do so, we set the crystal field splitting to a negative value at one site $i_{2}=\frac{L}{2}$ near the center of an $\mathrm{OBC}$ chain. In the ground state, orbital 2 thus has one electron at site $i_{2}$ and the AFM state in orbital 1 has at least one domain wall, which can sit either around site $i_{2}$ or at the open ends. At $J_{H}=0$, both positions have equal weight. In the presence of $J_{H}>0$, the preferred position depends on total $S^{z}=\frac{1}{2}\left(N_{\uparrow}-N_{\uparrow}\right)$ : For $S^{z}=1$ (describing the case with a spin flip) the domain wall is found predominantly around $i_{2}$ while it prefers the open chain ends for $S^{z}=0$, indicating attraction resp. repulsion. Numerical spectra in Fig. 1 do not show bound states, but the build-up of spectral weight at lower (higher) excitation energies can be explained by including such a spinon-holon interaction into a phenomenological description 23 in terms of spinon and holon.

\section{Conclusions.}

Fractionalization of the electron into spin and charge has long been realized as an intriguing property of onedimensional systems. The question of interactions between the fractionalized parts then naturally arises, even if they are not be strong enough to glue the electron back together. We find here that orbital excitations can offer insights into this aspect of spin-charge separation that are not easily accessible to one-particle excitations.

Orbital excitations had been shown to exhibit spinorbit separation in analogy to spin-charge separation, with the orbiton taking the role of the holon. We have here seen that Hund's rule leads to an attraction or repulsion between spinon and orbiton, depending on whether the excitation includes a spin flip or not. Their microscopic origin can be understood as a dynamic signature of the Goodenough-Kanamori rules that favor FM (AFM) spins on bonds with different (identical) orbitals. Hund'srule-induced interactions are not strong enough to induce (anti-)bound states, but they lead to clear signatures by shifting spectral weight to the upper resp. lower edge of the one-spinon-one-orbiton part of the spectrum.

We thank K. Wohlfeld for stimulating discussions and careful reading of the manuscript. This research was supported by the Deutsche Forschungsgemeinschaft, via the Emmy-Noether program (DA 1235/1-1) and FOR1807 (DA 1235/5-1). 
[1] P. W. Anderson, Physics Today 50, 42 (1997)

[2] E. H. Lieb and F. Y. Wu, Phys. Rev. Lett. 20, 1445 (1968)

[3] T. Giamarchi, Quantum Physics in One Dimension (Oxford University Press, Oxford, 2003).

[4] C. Kim, A. Y. Matsuura, Z.-X. Shen, N. Motoyama, H. Eisaki, S. Uchida, T. Tohyama, and S. Maekawa, Phys. Rev. Lett. 77, 4054 (1996)

[5] B. J. Kim, H. Koh, E. Rotenberg, S.-J. Oh, H. Eisaki, N. Motoyama, S. Uchida, T. Tohyama, S. Maekawa, Z.X. Shen, and C. Kim, Nature Physics 2, 397401 (2006)

[6] K. Wohlfeld, M. Daghofer, S. Nishimoto, G. Khaliullin, and J. van den Brink, Phys. Rev. Lett. 107, 147201 (2011)

[7] J. Schlappa, K. Wohlfeld, K. J. Zhou, M. Mourigal, M. W. Haverkort, V. N. Strocov, L. Hozoi, C. Monney, S. Nishimoto, S. Singh, A. Revcolevschi, J. S. Caux, L. Patthey, H. M. Rønnow, J. van den Brink, and T. Schmitt, Nature 485, $82(2012)$.

[8] C.-C. Chen, M. van Veenendaal, T. P. Devereaux, and K. Wohlfeld, Physical Review B 91, 165102 (2015)

[9] B. A. Bernevig, D. Giuliano, and R. B. Laughlin, Phys. Rev. Lett. 87, 177206 (2001)

[10] M. Greiter and D. Schuricht, Phys. Rev. B 71, 224424 (2005)

[11] B. A. Bernevig, D. Giuliano, and R. B. Laughlin, Phys. Rev. Lett. 96, 059702 (2006)

[12] J. Smakov, A. L. Chernyshev, and S. R. White, Phys. Rev. Lett. 98, 266401 (2007).

[13] K. I. Kugel and D. I. Khomskii, Soviet Physics Uspekhi 25, 231 (1982).

[14] M. M. Sala, V. Bisogni, C. Aruta, G. Balestrino, H. Berger, N. B. Brookes, G. M. de Luca, D. D. Castro, M. Grioni, M. Guarise, P. G. Medaglia, F. M. Gra- nozio, M. Minola, P. Perna, M. Radovic, M. Salluzzo, T. Schmitt, K. J. Zhou, L. Braicovich, and G. Ghiringhelli, New Journal of Physics 13, 043026 (2011).

[15] L. J. P. Ament, M. van Veenendaal, T. P. Devereaux, J. P. Hill, and J. van den Brink, Rev. Mod. Phys. 83, 705 (2011)

[16] J. Kim, D. Casa, M. Upton, T. Gog, Y.-J. Kim, J. Mitchell, M. Van Veenendaal, M. Daghofer, J. van den Brink, G. Khaliullin, and B. Kim, Phys. Rev. Lett. 108, $177003(2012)$

[17] K. Wohlfeld, S. Nishimoto, M. W. Haverkort, and J. van den Brink, Phys. Rev. B 88, 195138 (2013).

[18] V. Bisogni, K. Wohlfeld, S. Nishimoto, C. Monney, J. Trinckauf, K. Zhou, R. Kraus, K. Koepernik, C. Sekar, V. Strocov, B. Büchner, T. Schmitt, J. van den Brink, and J. Geck, Phys. Rev. Lett. 114, 096402 (2015)

[19] J. Kim, M. Daghofer, A. H. Said, T. Gog, J. van den Brink, G. Khaliullin, and B. J. Kim, Nature Communications 5, 4453 (2014)

[20] E. M. Plotnikova, M. Daghofer, J. van den Brink, and K. Wohlfeld, Phys. Rev. Lett. 116, 106401 (2016)

[21] Z. N. C. Ha and F. D. M. Haldane, Phys. Rev. Lett. 73, 2887 (1994)

[22] Y. Kato, Phys. Rev. Lett. 81, 5402 (1998)

[23] H. Suzuura and N. Nagaosa, Phys. Rev. B 56, 3548 (1997)

[24] J. S. Griffith, The Theory of Transition Metal Ions (Cambridge University Press, Cambridge, 1971).

[25] C. Castellani, C. Natoli, and J. Ranninger, Phys. Rev. B 18, 4945 (1978)

[26] A. S. Ovchinnikov, I. G. Bostrem, and V. E. Sinitsyn, Theoretical and Mathematical Physics 162, 179 (2010).

[27] B. Kumar, Phys. Rev. B 87, 195105 (2013).

[28] J. B. Goodenough, Phys. Rev. 100, 564 (1955).

[29] J. Kanamori, Journal of Physics and Chemistry of Solids 10, 87 (1959)

[30] S. Sorella and A. Parola, Phys. Rev. B 57, 6444 (1998) 